\title{
Thyroxine restores severely impaired cutaneous re-epithelialisation and angiogenesis in a novel preclinical assay for studying human skin wound healing under "pathological" conditions ex vivo
}

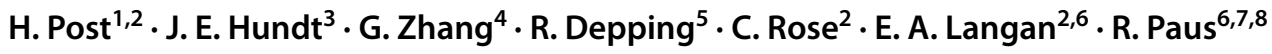 \\ Received: 22 May 2019 / Revised: 14 February 2020 / Accepted: 28 March 2020 / Published online: 22 June 2020 \\ (c) The Author(s) 2020
}

\begin{abstract}
Impaired cutaneous wound healing remains a major healthcare challenge. The enormity of this challenge is compounded by the lack of preclinical human skin wound healing models that recapitulate selected key factors underlying impaired healing, namely hypoxia/poor tissue perfusion, oxidative damage, defective innervation, and hyperglycaemia. Since organ-cultured human skin already represents a denervated and impaired perfusion state, we sought to further mimic "pathological" wound healing conditions by culturing experimentally wounded, healthy full-thickness frontotemporal skin from three healthy female subjects for three days in either serum-free supplemented Williams' E medium or in unsupplemented medium under "pathological" conditions (i.e. hypoxia [5\% $\left.\mathrm{O}_{2}\right]$, oxidative damage $\left[10 \mathrm{mM} \mathrm{H}_{2} \mathrm{O}_{2}\right.$ ], absence of insulin, excess glucose). Under these "pathological" conditions, dermal-epidermal split formation and dyskeratosis were prominent in organ-cultured human skin, and epidermal reepithelialisation was significantly impaired $(p<0.001)$, associated with reduced keratinocyte proliferation $(p<0.001)$, cytokeratin 6 expression $(p<0.001)$ and increased apoptosis $(p<0.001)$. Moreover, markers of intracutaneous angiogenesis (CD31 immunoreactivity and the number of of CD31 positive cells and CD31 positive vessel lumina) were significantly reduced. Since we had previously shown that thyroxine promotes wound healing in healthy human skin ex vivo, we tested whether this in principle also occurs under "pathological" wound healing conditions. Indeed, thyroxine administration sufficed to rescue re-epithelialisation $(p<0.001)$ and promoted both epidermal keratinocyte proliferation $(p<0.01)$ and angiogenesis in terms of CD31 immunoreactivity and CD31 positive cells under "pathological" conditions $(p<0.001)$ ex vivo. This demonstrates the utility of this pragmatic short-term ex vivo model, which recapitulates some key parameters of impaired human skin wound healing, for the preclinical identification of promising wound healing promoters.
\end{abstract}

Keywords Wound healing $\cdot$ Hypoxia $\cdot$ Skin model $\cdot$ Ex vivo

H. Post and J. E. Hundt have contributed equally to this work as first authors.

E. A. Langan and R. Paus have contributed equally to this work as senior authors.

Electronic supplementary material The online version of this article (https://doi.org/10.1007/s00403-020-02092-z) contains supplementary material, which is available to authorized users.

\section{R. Paus}

rxp803@med.miami.edu

Extended author information available on the last page of the article

\section{Introduction}

Chronic skin ulceration, particularly in the context of an ageing population, is a complex challenge in daily medical practice and is associated with (i) significant morbidity, (ii) impaired quality of life and (iii) substantial health care costs [1]. Indeed, in Germany alone, the estimated annual costs associated with the treatment of leg ulceration reach almost 9000 Euros (=9900 USD) per patient. With approximately 330,000 patients being treated annually, the result is an annual cost of approximately 3 billion Euros ( 3.3 billion USD) $[25,26]$. Leg ulcer management costs in the U.S. are considerably higher and are expected to increase further, not least due to the epidemic-like increase in diabetic foot ulcers [27]. 
Effective wound healing (WH) is a metabolically demanding process that relies upon the co-ordinated action of multiple tightly regulated complex biological processes, such as the timely initiation and termination of inflammation, adequate cell proliferation and efficient tissue remodelling, all of which are dependent upon sufficient tissue oxygenation, energy supply and innervation [17, 18, 22, 62]. Dysregulation of the WH process, at any level, may result in failure to re-establish tissue integrity, culminating in the development of a chronic wound $[17,18]$. Moreover, co-morbidities such as diabetes mellitus, obesity and peripheral vascular disease not only contribute to the development of chronic wounds but may also hinder their clinical resolution [38].

Despite the presence of a multitude of both in vivo [20, $61,69]$ and in vitro [48, 49, 59, 67] WH models, the development of optimally suited preclinical WH research models that not only sufficiently reflect in vivo WH conditions in patients with multiple chronic co-morbidities but which can also predict WH-promoting agents remains a key challenge in the field of cutaneous WH research [45]. Instead, research efforts have focussed on the development of sophisticated murine WH models, including diabetic mice models [42], in which the effects of specific diseases on WH can be investigated. However, given the recognised differences between cutaneous WH in mice and humans, the clinical relevance of these models to human $\mathrm{WH}$ is, at best, limited.

Furthermore, the available human WH models typically lack most resident skin cell populations and skin appendages, for example, the hair follicle (HF) $[2,16]$ and, therefore, fail to represent full "skin equivalents." Experimentally wounded human skin organ culture models [41, 43, 73] contain all of key resident cutaneous cell populations, native extracellular matrix whose WH-facilitating functions are very challenging to reconstitute [50] and skin appendages as well as a structurally intact, though no longer perfused, skin vasculature. Therefore, human skin organ culture systems are not only closest to the clinical in vivo situation but also constitutively recapitulate two important aspects of impaired $\mathrm{WH}[30,33,75]$ in that they are non-perfused and functionally denervated [74]. Using serum-free culture conditions $[37,41,75]$, these ex vivo WH models can be easily standardised, optimising reproducibility, and circumventing the confounding influences of various serum-dependent WHmodulatory factors [75]. Furthermore, these models can be utilised to investigate the aspects of angiogenesis, central to effective WH $[34,74]$.

However, even these models often fail to recapitulate the factors complicating WH in vivo, namely hypoxia, oxidative stress and hyperglycaemia. Therefore, based on previously published full-thickness human skin WH ex vivo models [31, 34, 37, 41, 74, 75], we sought to further simulate "pathological" WH conditions typically found under healing-impaired conditions, namely hyperglycaemia, hypoinsulinaemia, hypoxia and oxidative stress, that may be found in diabetic leg ulcers, by applying this to short-term organ-cultured human skin. Given the evidence that the HF may accelerate $\mathrm{WH}$ and that it may be hair cycle dependent $[2,28,39]$, we specifically sought to establish and validate our model using skin rich in terminal HFs, namely frontotemporal scalp skin.

In the second part of our study, we sought to ascertain the extent to which an agent known to promote WH in the human full-thickness skin ex vivo WH model, namely thyroxine (T4), also exerts WH-promoting effects in the "pathological" WH model. For this, we chose to test T4 as the lead compound, because it has been shown to promote cutaneous WH both in mice in vivo $[53,54]$ and in healthy human skin ex vivo [74], and has an extensively characterised toxicological profile in humans [32]. Indeed, this proof-of-principle part of the study was designed to determine whether the model could preclinically identify candidate WH-promoting agents, which exerted their effects despite conditions known to profoundly impair $\mathrm{WH}$.

\section{Materials and methods}

\section{Organ culture of experimentally wounded human skin under "standard" and "pathological" healing conditions}

Temporal and occipital region scalp skin samples from six females (aged 52-65 years) were obtained as by-products from cosmetic surgical procedures. All patients provided informed consent, and the study was approved by the Institutional Research Ethics Committee at the University of Luebeck (University of Luebeck, license: Reference 06-109). All experiments were performed in accordance with the ethical standards set by the Declaration of Helsinki and its later amendments.

Skin samples from the first three patients were used to establish a "pathological" WH model and skin samples from the remaining subjects were used to test the effects of T4, under standard and "pathological" conditions (see below), on several WH-related parameters. Skin fragments were "wounded" via a 4-mm punch biopsy, with the tissue periphery, which rapidly shows signs of epiboly, i.e. the tendency of the epithelium to rapidly enclose traumatically exposed mesenchyme, $[10,58]$ taken as the "wound edge". This approach was in contrast to the established punch-ina-punch design used to examine skin wounding ex vivo [41, 43] and was necessary given the extensive dermal-epidermal separation which occurred under "pathological" conditions.

The punch biopsies were then placed in standard supplemented Williams' E medium (WE) (22), or in the modified "pathological" medium. All punches were cultured in 
a six-well plate, each well containing $3 \mathrm{ml}$ of medium and two skin punches, floating at the liquid-air interface. After completion of the organ culture, skin punches were snap frozen in cryomatrix and liquid nitrogen and stored at $-80{ }^{\circ} \mathrm{C}$ until cryosections were cut, stained and analysed. See Supplementary Fig. 1 for the experimental setup.

\section{"Standard" versus "pathological" skin organ culture medium}

Serum-free WE supplemented with l-glutamine ( $2 \mathrm{mmol} / \mathrm{l})$, hydrocortisone $(10 \mathrm{ng} / \mathrm{ml})$, insulin $(10 \mu \mathrm{g} / \mathrm{ml})$ and streptomycin $(10 \mu \mathrm{g} / \mathrm{ml}) /$ penicillin $\mathrm{G}(100 \mathrm{IU} / \mathrm{ml})$ served as the standard control culture medium $[8,37,41]$. Hydrocortisone is a necessary supplement of serum-free medium for ex vivo skin organ culture. The supplemented WE medium allows human skin to be cultured for up to two weeks [37].
The "pathological" WH conditions included hyperglycaemia, achieved by the addition of glucose. Given that diabetes can be diagnosed with a random serum glucose concentration of $\geq 11.1 \mathrm{mmol} / \mathrm{dl}$ ( $\geq 200 \mathrm{mg} / \mathrm{dl}$ ), and that tissue damage due to hyperglycaemia is a long-term complication [4, 55], we selected a glucose concentration ten times of that used to diagnose diabetes to ensure an adequate effect in our short-term model. Given the pharmacological concentrations of glucose used to severely impair WH in short-term organ culture (which rather imitate hyperglycemic diabetic coma conditions than classical diabetes mellitus-associated chronic skin damage associated with peripheral neuropathy and diabetic microangiopathy), it is important to point out that we did not seek to fully simulate diabetic conditions ex vivo.

Hyperglycaemia results in the formation of advanced glycation end-products (AGEs). The formation of AGEs is also (a) PAS

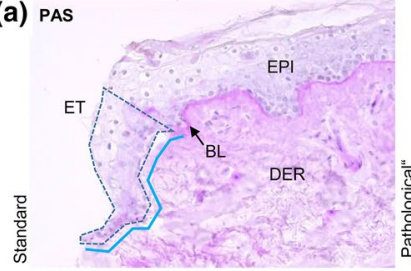

(c) H\&E

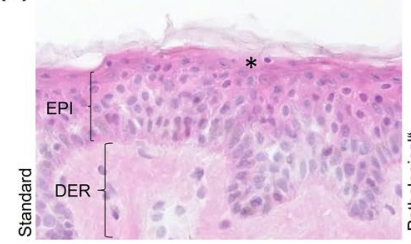

(e) PAs

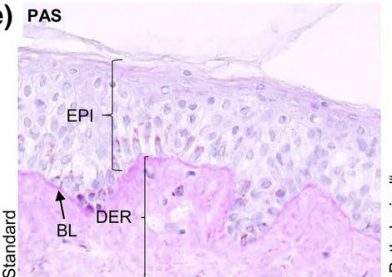

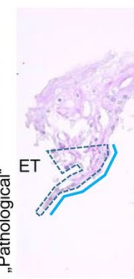
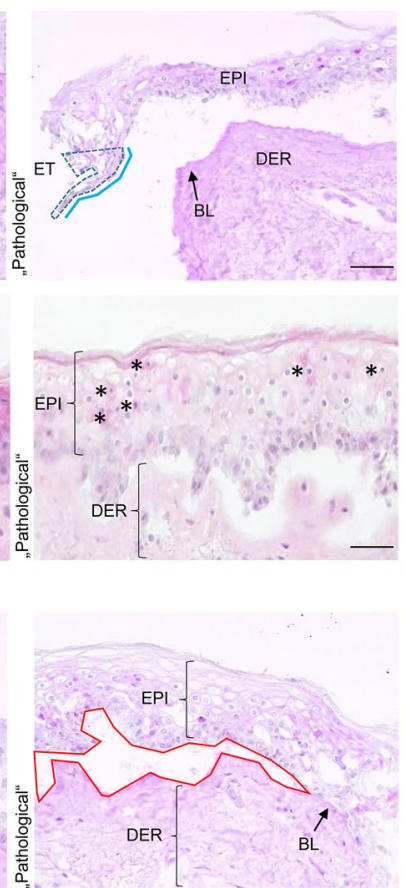

(b)

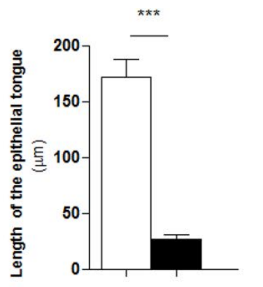

(d)

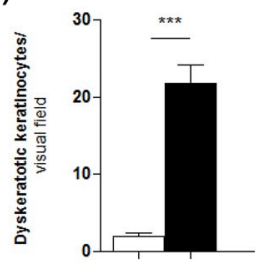

(f)

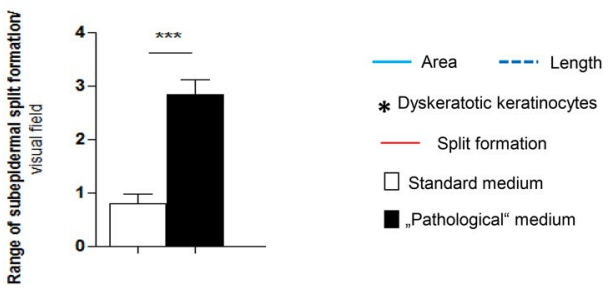

Fig. 1 "Pathological" culture conditions resulted in major changes in epithelial morphology and severe reduction of epithelial tongue formation. a Periodic acid-Schiff (PAS) staining of left wound edges with a physiologically developed epithelial tongue (ET) under standard conditions and the smaller ET under "pathological" conditions. Evaluations were performed by measuring the marking of the length (light blue continuous line) and the area (dark blue dashed line). b Comparing the sizes (length and area) of the ETs from standard and "pathological" conditions revealed dramatic differences under "pathological" conditions. $* * * p \leq 0.001$; pooled data from three different patients, six punches, 26-29 skin sections; mean \pm SEM; $p$ value was calculated by Mann-Whitney U test for unpaired samples. c Haematoxylin and Eosin staining of skin punches from standard and "pathological" culture conditions showing the epidermis and labelled dyskeratotic keratinocytes (KCs) (black stars). d Dyskeratotic KCs were counted inside the whole epidermis per visual field. Dyskeratosis was a predominant phenomenon under "pathological" culture conditions. $* * * p \leq 0.001$; pooled data from three different patients, 5-6 punches, 23-44 skin sections; mean \pm SEM; $p$ value was calculated by MannWhitney $U$ test for unpaired samples. e PAS staining represents the different manifestations of split formation (red continuous line) comparing standard and "pathological" cultured skin. f The extent of split formation was assessed by a semi-quantitative method per visual field, and it was significantly higher detectable in skin of "pathological" culture conditions. ${ }^{* * *} p \leq 0.001$; pooled data from three different patients, six punches, 38-44 skin sections; mean \pm SEM; $p$ value was calculated by Mann-Whitney $U$ test for unpaired samples. ET epithelial tongue, EPI: epidermis, DER: dermis, $B L$ : basal lamina. Scale bars: $50 \mu \mathrm{m}$ 
promoted by oxidative stress and leads to tissue dysfunction by the cross-linking of collagens, activation of macrophages and covalent bonding of lipoproteins [57]. Under "standarddiabetic" conditions of latent hyperglycaemia, the formation of AGEs takes several weeks [4] and were, therefore, unsuitable for our model. Profound over-supply of substrates can accelerate this process to a duration of only a few hours [55]. Accumulation of AGEs is one of the main pathomechanisms causing tissue damage in diabetic patients. Additionally, matrix glycosylation was identified as an underlying mechanism of an increased cellular apoptosis, inhibition of cell proliferation, migration and angiogenesis in the skin of diabetic patients [15]. Therefore, we applied a concentration of $138.8 \mathrm{mM}$ $(2500 \mathrm{mg} / \mathrm{dl})$ of glucose to create hyperglycaemic circumstances. Importantly, this very high glucose serum concentration is pathologically relevant as it can be reached in patients undergoing hyperglycaemic coma. In addition, insulin was omitted to imitate hypoinsulinemic diabetic-like conditions.

Oxidative damage was experimentally induced by adding $3.06 \mu \mathrm{l}$ of hydrogen peroxide $\left(\mathrm{H}_{2} \mathrm{O}_{2}\right)$ per well (concentration $10 \mathrm{mM}$ ) based on our prior experience with experimental induction of oxidative damage in human skin appendages $[24,72]$. Finally, hypoxia was generated by incubation under $5 \%$ oxygen, using a hypoxia incubator (Ruskinn InVivo400) [9] instead of the standard oxygen supply of $20.9 \%$ in ambient air.

To determine whether the "pathological" model is capable of identifying candidate agents that promote human skin WH even under severely impaired WH conditions ex vivo, we tested $\mathrm{T} 4$ as the lead compound since this peptide hormone promotes skin WH, both in mice in vivo $[53,54]$ and in healthy human skin ex vivo [74].

Test groups were supplemented with $\mathrm{T} 4$ at a concentration of $100 \mathrm{nM}$ to promote mitochondrial activity and to reduce standard ageing-associated read out parameters in human skin ex vivo [65]; this T4 concentration also promotes mitochondrial activity and biogenesis in human scalp HF [66]. Moreover, we had previously shown that $100 \mathrm{nM}$ $\mathrm{T} 4$ enhances hair matrix keratinocyte $(\mathrm{KC})$ proliferation and prolongs anagen hair growth [64], both of which are expected to exert overall WH-promoting effects [2, 39]. Given that the very limited availability of human skin for organ culture precluded running a dose-response study, $100 \mathrm{nM}$ T4 appeared to be an optimally chosen test concentration. This concentration could also be easily reached after topical application under clinical conditions.

\section{Staining methods and quantitative (immuno-) histomorphometry}

Phenotypic changes in the subcorneal epidermis were quantified by counting the total number of cells demonstrating the morphological features of dyskeratosis, i.e. premature keratinisation with a pyknotic, hyperchromatic nucleus and lightened cytoplasm ("halo") [46]. Dyskeratotic cells were recognized by routine haematoxylin and eosin (H\&E) histochemistry. The extent of subepidermal split formation was evaluated by determining the degree of separation along the entire epidermis. We assessed re-epithelialisation by quantifying the length and area of the neoepidermis ["epithelial tongue" (ET)] on day 3 as previously described $[31,34,74]$. Quantitative histomorphometry for split formation and reepithelialisation was performed after histochemical Periodic acid-Schiff (PAS) staining to facilitate demarcation of the basal lamina (BL) zone, also in the newly formed neoepidermis [41]. For each parameter, we selected pre-defined areas for the evaluations as follows: dyskeratosis and the dermal-epidermal split formation were measured along the skin punch. The length and area of the epithelial tongue was measured in two visual fields (both edges) and the number of Ki-67/TUNEL-positive cells inside the epithelial tongues was measured in addition to the number of Ki-67/TUNELpositive cells in three additional visual fields. Cytokeratin 6 (CK6) immunoreactivity (IR) was measured in the entire epidermis skin punch and the angiogenesis parameters were measured in the dermis adjacent to the ETs and in one visual field in the middle of the skin punch.

Evaluation was performed with Image $\mathbf{J}$ software by measuring the length and area of the ET. Photomicrographs were obtained using the Keyence-Biozero 8000. Dimensions were orientated via the insertion of a $50 \mu \mathrm{m}$ scale bar. Immunofluorescence (IF) microscopy for the employed read-out parameters (Ki-67/ TUNEL, CK6 and CD31) was performed and analysed as described previously [37, 41].

Proliferation and apoptosis were determined by Ki-67/ TUNEL double-IF staining. The total number of Ki-67, TUNEL and DAPI was counted in a defined area inside the epidermis and ETs. In the first experimental set-up, Ki-67 positive and TUNEL positive cells were counted inside a defined area of the ET but also in three additionally defined areas of epidermis. In the second experimental setup (i.e. addition of T4), evaluation of apoptosis and proliferation was restricted to the ETs.

CK6 expression was measured given that it is increased in both hyperproliferative and wounded epithelium $[52,70]$ and given that a thyroid hormone $(\mathrm{TH})$ response element is located in the promoter region of the CK6 gene [71].

Angiogenesis, another key WH parameter, was assessed by the detection of CD31 positive cells, a validated endothelial cell marker [40, 74]. Three angiogenesis parameters were analysed, namely (i) CD31 immunoreactivity (IR), (ii) the total number of CD31positive nuclei and (iii) the total number of CD31 positive lumina. All three criteria were analysed in two predefined dermal visual fields directly underneath the neoepithelial tongue. CK6 and CD31 IR were normalised to the control group $[41,74]$. The antibodies used, and 
their concentrations, are available in supplementary Tables 1 and 2. All pictures were analysed with Image $\mathbf{J}$ software (National Institutes of Health, Bethesda, MD).

\section{Statistical analyses}

All data sets were tested for normal distribution using Graph Pad Prism v. 3.00 (Graph-Pad Software Chicago, IL, USA). When the data set was normally distributed, the $t$-test was used when comparing two groups. When the data were not normally distributed, the Mann-Whitney $U$ test was selected. Statistical analyses were also performed using Graph Pad Prism v. 3.00 (Graph-Pad Software Chicago, IL, USA). All data were expressed as mean \pm SEM (standard error of the mean); $p$ values $*<0.05, * * \leq 0.01, * * * \leq 0.001$ were regarded significant. For each parameter, two punches were cultured and compared. All data were pooled, resulting in a total of 19-54 skin fragments per experimental group, derived from three different female patients to establish the model and from three female patients to test the effect of thyroxine. Data pooling was justified since the obtained results in at least three independently run experiments showed comparable trends.

\section{Results}

\section{"Pathological" wound healing culture conditions induce characteristic morphological changes including dyskeratosis, dermal-epidermal separation and impaired re-epithelialisation}

First, we tested the working hypothesis that "pathological" WH conditions would negatively effect skin structure and integrity as well as re-epithelialisation, even in shortterm organ culture. After wounding, KCs migrate from the $\mathrm{BL}$ along the wound edge to restore the epithelial barrier after wounding, [18] which can be assessed qualitatively and quantitatively by measuring the formation of ETs, i.e. neoepidermis formation [41, 74]. Indeed, "pathological" WH conditions almost completely prevented this process (Fig. 1a, b). Importantly, however, at least some residual reepithelialisation capacity was preserved.

Moreover, the number of intraepidermal dyskeratotic cells and the extent of the epidermal separation from the BL(epidermolysis) were both significantly increased in the skin under "pathological" WH conditions compared to standard human skin organ culture conditions (Fig. 1c-f). This likely reflected severe tissue damage resulting from metabolic, osmotic and oxidative stress since increased cell size, nuclear shifting and vacuolation of KCs during tissue hypoxia have been previously described under these conditions [60].
"Pathological" wound healing conditions induce apoptosis and inhibit proliferation and cytokeratin 6 expression of epidermal keratinocytes ex vivo

Next, we examined how "pathological" WH conditions influenced epidermal $\mathrm{KC}$ apoptosis and proliferation ex vivo. KC apoptosis was significantly increased throughout the entire epidermis under "pathological" conditions compared to that in control conditions (Fig. 2a, b). Moreover, the "pathological" conditions resulted in decreased KC proliferation, in line with evidence of severely reduced cell viability and the disruption of epidermal morphology and epithelial migration shown in Fig. 1. However, substantial residual proliferative $\mathrm{KC}$ activity was still present even under these conditions, attesting to sustained epidermal viability after 3 days of organ culture even under these severely impaired $\mathrm{WH}$ conditions ex vivo.

Given that CK6 protein expression is strongly upregulated in wounded human epidermis and during epidermal regeneration [41] and that this keratin actively impacts KC migration during wound healing [52], we also tested whether "pathological" medium conditions also affect CK6 expression. Indeed, CK6 IR, measured in the ET and epidermis, was decreased under "pathological" WH conditions (Fig. 2c). This further attests to the WH-impaired status of experimentally wounded human epidermis under these assay conditions.

\section{Angiogenesis is impaired under "pathological" wound healing conditions}

To complete the characterisation of our "pathological" WH model, we asked whether hyperglycaemia, hypoinsulinaemia, hypoxia and oxidative stress also impact upon angiogenesis since we had had previously shown that indications of angiogenesis are still visible, albeit a fluctuating levels, even after several days of organ-culturing human skin under serum-free conditions [34, 74]. Markers of angiogenesis were significantly downregulated under "pathological" medium conditions, as measured by assessing (i) total CD31 immunoreactivity, (ii) the number of CD31 + endothelial cells, and (iii) the number of CD31 positive blood vessel cross sections (i.e. with a discernible vessel lumen) per visual field (Fig. 2d-i).

In summary, these findings demonstrate that the simple, easily set up organ culture system for experimentally wounded skin reported here rapidly generates severely healing-impaired previously healthy human skin within just three days ex vivo. Dyskeratosis and split formation were measured to confirm that the skin was being sufficiently stressed by the "pathological" conditions. Having confirmed this, we next addressed the ability of T4 to influence the 
(a)

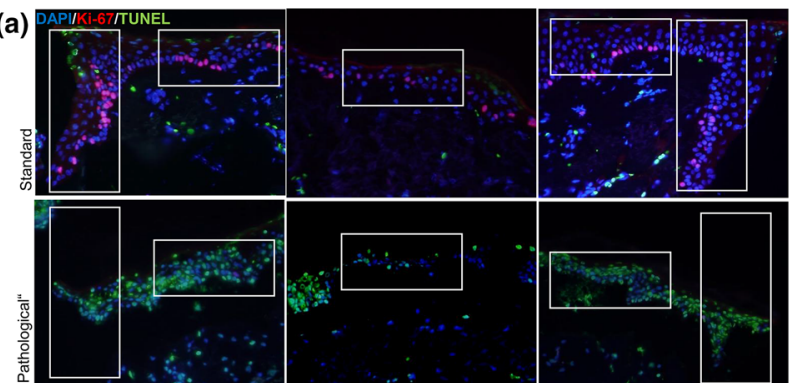

(b)

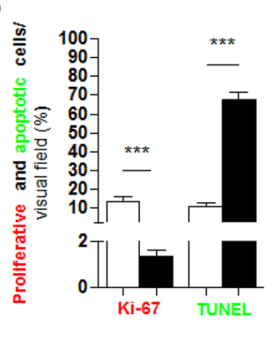

(c)

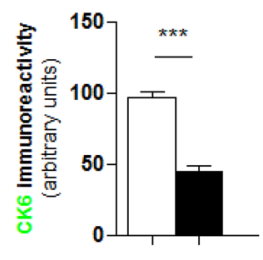

(d)

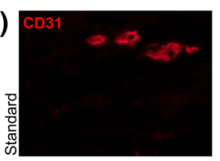

(e)

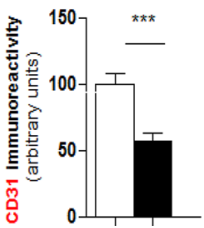

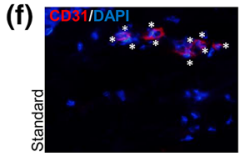

(g)

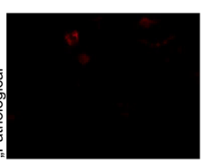

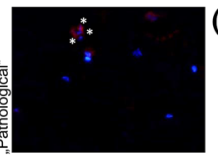

***

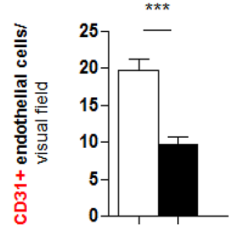

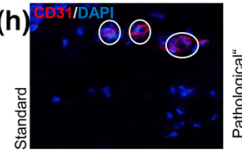

(i)

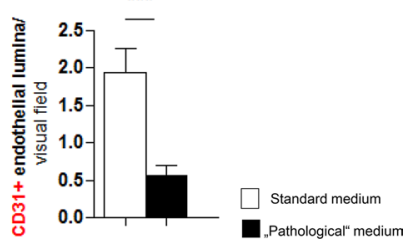

Fig. 2 The effect of "pathological" medium conditions on keratinocyte proliferation, cytokeratin 6 and neoangiogenesis. a Double immunofluorescent Ki-67/TUNEL/DAPI staining of left and right epithelial tongues (ETs) and the epidermis. Red fluorescent immunoreactivity (IR) represents Ki-67 positive (proliferating), green fluorescent IR represents TUNEL positive (apoptotic) and blue fluorescent IR represents DAPI positive (all nuclei) which were counted separately in defined reference areas (white boxes). b The amount of proliferative and apoptotic cells is provided as percentages of all cells (number of DAPI-positive cells was set as $100 \%$ ) inside the reference area from the ETs and epidermis. The number of proliferative cells is significantly lower and apoptosis is highly increased under "pathological" conditions. ${ }^{* * *} p \leq 0.001$; pooled data from three different patients, six punches, $31-35$ skin sections; mean \pm SEM; $p$ value was calculated by Mann-Whitney $U$ test for unpaired samples. c Cytokeratin 6 (CK6) protein expression in the "pathological" cultured skin was significantly reduced compared to normalised results of the standard medium $(=100 \%)$. $* * * p \leq 0.001$; pooled data from three different patients, six punches, 34-35 skin sections; mean \pm SEM; $p$ value was calculated by Mann-Whitney $U$ test for unpaired samples. $\mathbf{d}$ and e Immunofluorescent CD31 staining of an analysed part of the dermis demonstrating a clear red fluorescent CD31-positive signal whilst this is hardly detectable in the skin of "pathological" medium

key parameters of $\mathrm{WH}$, namely re-epithelialisation and neoangiogenesis under "pathological" conditions.

\section{Thyroxine promotes the proliferation and Cytokeratin 6 expression of human epidermal keratinocytes, stimulates angiogenesis, and markedly reduces keratinocyte apoptosis ex vivo}

Most WH assays employed in vitro, ex vivo, or in vivo examine the impact of test agents and experimental manipulations under WH conditions that reflect acute WH in essentially healthy tissue. Unsurprisingly, WH-promoting effects conditions. The immunoreactivity (IR) was assessed per visual field of three defined dermal reference areas comparing "pathological" medium conditions to normalised results of the standard medium conditions $(=100 \%)$. CD31 IR was significantly less detected in the "pathological" sections. f and $\mathbf{g}$ An endothelial cell was counted for each blue fluorescent DAPI-positive nucleus associated with a red fluorescent CD31-positive signal (white stars) which were sparsely found under "pathological" medium conditions. The number of endothelial cells was assessed per visual field of three defined dermal reference areas comparing the results of "pathological" medium conditions to the results of standard medium conditions. The number of endothelial cells was significantly lower under "pathological" conditions. Immunofluorescent CD31/DAPI staining of a defined dermal area. An endothelial lumen (white encircled) was counted for bluefluorescent DAPI-positive nuclei associated with a red-fluorescent CD31-positive signal forming a lumen. The number of lumina was assessed per visual field of three defined dermal reference areas comparing the results of "pathological" medium conditions to the results of standard medium conditions. $\mathbf{h}$ and $\mathbf{i}$ The number of lumina was significantly lower under "pathological" conditions. Scale bar: $50 \mu \mathrm{m}$; $* * * p \leq 0.001$; pooled data from three different patients, six punches, 41-42 skin sections; mean $\pm \mathrm{SEM} ; p$ value was calculated by MannWhitney $U$ test for unpaired samples

seen under these more or less physiological conditions often do not translate into tangible clinical benefits under the only WH conditions that really matter in clinical practice: impaired WH and ulcer formation [45]. Therefore, the litmus test for any candidate $\mathrm{WH}$-promoting agent or treatment strategy is that it unfolds significant WH benefits, such as the acceleration and improvement of epidermal repair and WH-associated angiogenesis, also under clinically relevant WH-impaired conditions.

We, therefore, explored whether or not the candidate WHpromoting thyroid hormone, T4 [54, 74], positively impacts epidermal repair and angiogenesis and sought to examine the effect of T4 during "pathological" conditions, given its 
documented WH-promoting effect, albeit ex vivo [19, 29, 54].

Interestingly, the addition of $100 \mathrm{nM} \mathrm{T} 4$ for 3 days to the medium dramatically reduced epidermal $\mathrm{KC}$ apoptosis under standard $(p<0.01)$ and under "pathological" $(0.001)$ conditions (Fig. 3) and also significantly increased $\mathrm{KC}$ proliferation under "pathological" conditions $(p<0.05)$ culture conditions ex vivo. Moreover, the inhibitory effects of these culture conditions on ET formation (reflecting epidermal $\mathrm{KC}$ migration and epidermal repair) were reversed by $\mathrm{T} 4$ supplementation (Supp. 1). In addition, T4 tended to restore the reduced CK6 expression in the ET under "pathological" WH conditions (Fig. 3c, d).

$\mathrm{T} 4$ addition to the medium resulted in both significantly increased CD31 IR and enhanced the number of CD31 positive cells under "pathological" conditions" (Fig. 2d-i). However, T4 did not significantly affect the number of blood vessel cross sections even though there was a statistically non-significant increase (Fig. 4a-f). Thus, while T4 appears to have restored some of the negative impact of the tested experimental "pathological" WH conditions on intracutaneous angiogenesis ex vivo, it remains unclear whether angiogenesis was really promoted.

\section{Discussion}

The development of novel WH-promoting treatments is hampered by the lack of clinically relevant WH models in general, and the lack of WH models replicating WH in patients with multiple co-morbidities (diabetes, peripheral vascular disease) in particular. Therefore, we sought to establish a robust and clinically relevant "pathological" WH model and determine the extent to which candidate WH-promoting
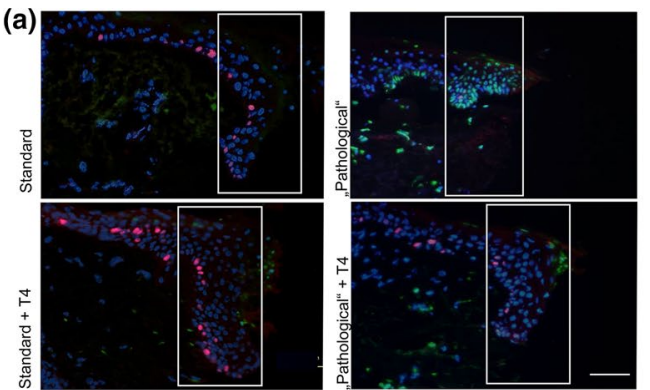

(b)

(c)

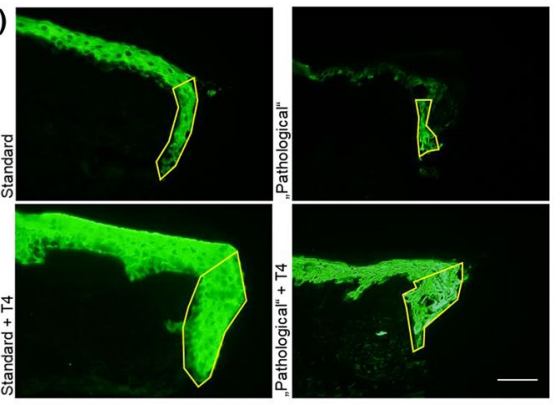

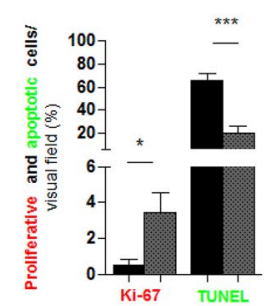

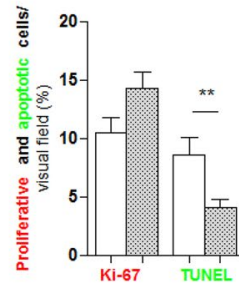

(d)

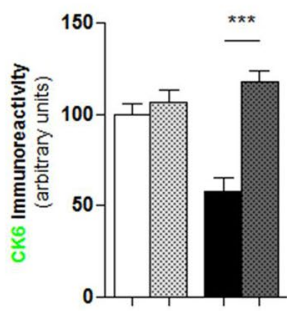

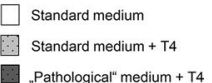

Pathological

„Pathological" medium
Fig. 3 Keratinocyte proliferation is increased by thyroxine (T4) and cytokeratin 6 (CK6) protein expression was reduced under "pathological" conditions and upregulated when T4 was added to the medium. a Double-immunofluorescent Ki-67/TUNEL/DAPI staining showing right wound edges and epidermal tongues (ETs). Statistical analyses of the amount of proliferative/apoptotic cells comparing standard versus (vs.) standard + T4 conditions and "pathological" vs. "pathological" $+\mathrm{T} 4$ conditions. Results of proliferative and apoptotic cells are provided as percentage of all cells (number of DAPI-positive cells was set as 100\%) inside the reference area from the ETs. b The amount of proliferative cells tended to be increased under standard + T4 medium conditions but was only significant inside the ETs of "pathological" + T4 medium conditions. Apoptosis was reduced under T4-treated medium conditions most significantly under "pathological" medium conditions. $* * * p \leq 0.001,{ }^{* *} p \leq 0.01, * p<0.05$; pooled data from three different patients, six punches, 24-35 skin sections; mean \pm SEM; $p$ value was calculated by Mann-Whitney $U$ test for unpaired samples. Scale bar: $50 \mu \mathrm{m}$. c Immunofluorescent CK6 staining of skin sections showing right wound edges and ETs. Green fluorescent immunoreactivity (IR) represents the CK6-positive signal and appears brighter in the skin from T4-supplemented medium conditions. The CK6-positive IR was assessed in the ETs (yellow continuous line) by quantitative immunofluorescence morphometry. d Statistical analyses demonstrate the assessment of the CK6 IR comparing standard versus (vs.) standard + T4 conditions and "pathological" vs. "pathological" + T4 conditions. All results were normalised to the standard medium $(=100 \%)$. Upregulation of CK6 IR was significant under "pathological" conditions. *** $p \leq 0.001$; pooled data from three different patients, six punches, 34-35 skin sections; mean \pm SEM; $p$ value was calculated by Mann-Whitney $U$ test for unpaired samples. Scale bar: $50 \mu \mathrm{m}$ 


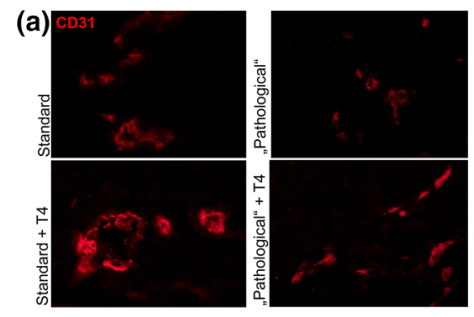

(d)

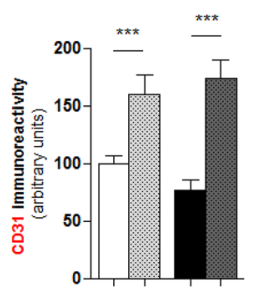

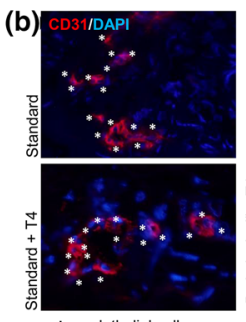

* endothelial cells

(e)

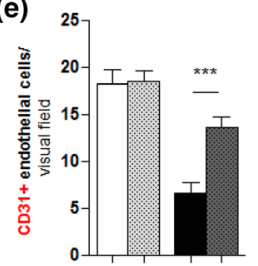

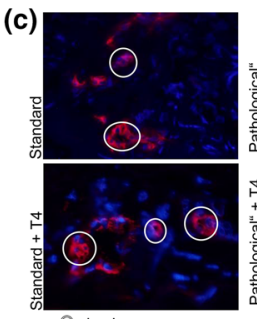

O lumina

(f)

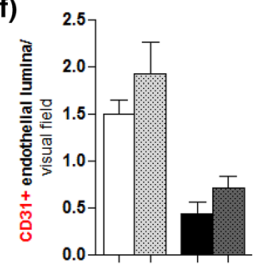

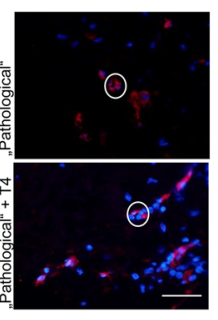

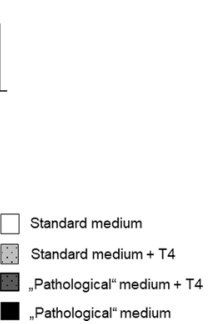

Fig. 4 Intracutaneous angiogenesis is strongly impaired but may be partially ameliorated by thyroxine (T4) under "pathological" conditions. a, b Immunofluorescent CD31 staining of an analysed part of the dermis demonstrating a brighter red-fluorescent CD31-positive signal in the skin of T4-supplemented medium conditions. Comparing the immunoreactivity (IR) of standard versus (vs.) standard +T4 conditions and "pathological" vs. "pathological" + T4 conditions normalised to the mean value of standard conditions to $100 \%$. The T4-supplemented groups show a significant upregulation of the CD31 positive IR which is even more obvious in "pathological" conditions. c, d Immunofluorescent CD31/DAPI staining of a defined dermal area. An endothelial cell was counted for each bluefluorescent DAPI-positive nucleus associated with a red-fluorescent CD31-positive signal (white stars). The number of endothelial cells was assessed per visual field of three defined dermal reference areas

agents could modulate WH under these carefully defined and reproducible conditions.

For example, hyperglycaemia and hypoinsulinaemia or insulin end-organ resistance are known to impair WH via a reduction in dermal fibroblast proliferation and migration. Furthermore, the low-grade inflammation and angiogenesis associated with diabetes and the metabolic syndrome, respectively, also contribute to impaired $\mathrm{WH}$ and tissue remodelling [21]. In addition, the chronic tissue hypoxia and the accumulation of $\mathrm{H}_{2} \mathrm{O}_{2}$ and reactive oxygen species (ROS), resulting from reduced perfusion in peripheral vascular disease, also disrupts WH in vivo [56].

Here we have developed and validated a pragmatic, welldefined, serum-free human skin organ culture model, in which the epidermal repair and angiogenesis can be studied under severely healing impaired ("pathological") WH conditions that mimic ex vivo, at least to some extent, the hypoxia, oxidative damage, impaired perfusion and innervation, hyperglycaemia and hypoinsulinaemia which may be present comparing the results of standard vs. standard $+\mathrm{T} 4$ conditions and "pathological" vs. "pathological" + T4 conditions. The number of CD31-positive endothelial cells was not significantly different comparing standard and standard $+\mathrm{T} 4$ but in the T4-supplemented "pathological" group, significantly higher than in the "pathological" untreated group. e, f Immunofluorescent CD31/DAPI staining of a defined dermal area. An endothelial lumen (white encircled) was counted for blue-fluorescent DAPI-positive nuclei associated to a redfluorescent CD31-positive signal forming a lumen. There was no significant difference of CD31-positive lumina, neither in the standard vs. standard + T4 nor in the "pathological" vs. "pathological" + T4 even though the T4-supplemented groups tended to contain a higher amount of lumina. Scale bar: $50 \mu \mathrm{m}$; $* * *<<0.001$; pooled data from three different patients, 39-54 skin sections; mean \pm SEM; MannWhitney $U$ test for unpaired samples

in diabetic ulcers in vivo. This model recreates impaired WH-associated alterations in $\mathrm{KC}$ morphology, reduced $\mathrm{KC}$ proliferation and migration, increased $\mathrm{KC}$ apoptosis, epidermolysis, and reduced angiogenesis and is, therefore, well suited for the preclinical testing of candidate WH-promoting agents that hope to positively affect these parameters. That T4 was able to partially "rescue" epidermal repair and promote angiogenesis, even under these severely healingimpaired clinically relevant WH conditions, in a human model, is interesting and potentially clinically relevant.

With the aim of validating our "pathological" WH model of human skin, we selected T4 as a candidate WH promotor based on its effects on mitochondrial biogenesis, KC proliferation and angiogenesis [36, 68]. The thyroid gland-derived hormone $\mathrm{T} 4$ is converted in peripheral tissues to the active hormone $\mathrm{T} 3$ and regulates metabolism. In terms of the skin, TH receptor (TH-R) is expressed in the human HF [64]; $\mathrm{TH}$-dependent signalling regulates the expression of selected keratins, can prolong the anagen phase of hair growth and 
stimulate hair matrix $\mathrm{KC}$ proliferation [47, 64]. It has also been reported that thyroid stimulating hormone (TSH) treatment promotes the proliferation of human epidermal KCs and dermal fibroblast [14]. Epidermal barrier function is maintained via upregulation of dermal TH-R [3]. Furthermore, TH reportedly stimulates angiogenesis in vivo [74].

The pro-angiogenic effect of $\mathrm{T} 4$ is mediated by the cell surface receptor integrin $\alpha v \beta 3$. Stimulation of this receptor on endothelial cells stimulates intracellular transcription of pro-angiogenic growth factors (GFs), like bFGF and VEGF [13].

Induced hypothyroidism in mice results in papillary rarefaction and could be treated via systemically applied triiodothyronine (T3) which induced sprouting angiogenesis in the heart tissue of the hypothyroid mice [12]. There is also evidence that $\mathrm{TH}$ can initialise angiogenesis in vitro using the "HUVEC"-model under serum-free medium conditions, supplemented by T4 $100 \mathrm{nM}$ [36].

Specifically, in terms of WH, T4 has been shown to have a WH-promoting effect compared to untreated groups in animal studies $[19,54]$. T3 enhanced $\mathrm{WH}$ processes in guinea pigs most likely by wound contraction [29]. T4 may represent an intriguing candidate WH promoter [7] especially if topical application can be delivered without affecting circulating T4 concentrations [11]. Additional potential WHpromoting agents, including interleukin 22 [6], pro-insulin C-peptide [35], and erythropoietin [5, 23] could also be preclinically tested in this clinically relevant WH model.

In terms of dissecting the molecular mechanism underlying the effect of $\mathrm{T} 4$ on $\mathrm{WH}$ in this model, future studies may wish to examine the extent to which fibroblast and HF stem cell proliferation contribute to the WH evidence in the model, for example, by determining matrix metalloproteinase, heat-shock protein and CK 15 expression, respectively. Indeed, therapies aimed at mobilising stem cells have recently be shown to promote cutaneous WH in diabetic rats [51]. Moreover, THs have been shown to not only regulate expression, apoptosis, and differentiation in human HF epithelial stem cells in situ and in vitro [63] but thyroid analogues also prolong the anagen phase of the hair cycle; a process intimately associated with WH $[2,44]$.

In summary, we report the development of a pragmatic organ culture assay that quickly transforms viable biopsywounded human skin into a "pathological" state, where WH is severely impaired. We have attempted to provide proof-ofprinciple that (i) human skin with severely "pathological" WH features is still capable of responding in a therapeutically desired manner to treatment with a candidate WH promoter and (ii) TH-R agonists are interesting, but as yet insufficiently investigated candidate promoters of "pathological" WH. This novel preclinical test system, which is characterised by severe hypoxia, excessive ROS levels, hyperglycaemia and insulin withdrawal, can serve as a surrogate assay for probing the efficiency of candidate $\mathrm{WH}$ promoters under well-standardized and clinically relevant ex vivo conditions.

Acknowledgements The authors gratefully acknowledge the excellent technical assistance of Claudia Kremling, Antje Winter-Keil, Nadine Merg and Elvira Jeske-Ehlers.

\section{Compliance with ethical standards}

Conflict of interest The authors state no conflict of interests, as this work was part of a strictly academic MD thesis project (of H.P., supervised by R.P.) that did not receive any industrial funding and did not lead to patent filing. For the record, R.P. is founder and managing owner of a company that also offers human skin wound-healing assays (www.monasteriumlab.com).

Open Access This article is licensed under a Creative Commons Attribution 4.0 International License, which permits use, sharing, adaptation, distribution and reproduction in any medium or format, as long as you give appropriate credit to the original author(s) and the source, provide a link to the Creative Commons licence, and indicate if changes were made. The images or other third party material in this article are included in the article's Creative Commons licence, unless indicated otherwise in a credit line to the material. If material is not included in the article's Creative Commons licence and your intended use is not permitted by statutory regulation or exceeds the permitted use, you will need to obtain permission directly from the copyright holder. To view a copy of this licence, visit http://creativecommons.org/licenses/by/4.0/.

\section{References}

1. Alavi A, Sibbald RG, Phillips TJ, Miller OF, Margolis DJ, Marston W, Woo K, Romanelli M, Kirsner RS (2016) What's new: management of venous leg ulcers: treating venous leg ulcers. J Am Acad Dermatol 74(4):643-664 (quiz 665-646)

2. Ansell DM, Kloepper JE, Thomason HA, Paus R, Hardman MJ (2011) Exploring the "hair growth-wound healing connection": anagen phase promotes wound re-epithelialization. J Invest Dermatol 131(2):518-528

3. Antonini D, Sibilio A, Dentice M, Missero C (2013) An Intimate relationship between thyroid hormone and skin: regulation of gene expression. Front Endocrinol (Lausanne) 4:104

4. Aronson D, Rayfield EJ (2002) How hyperglycemia promotes atherosclerosis: molecular mechanisms. Cardiovasc Diabetol 1:1

5. Arslantas MK, Arslantas R, Tozan EN (2015) Effects of systemic erythropoietin on ischemic wound healing in rats. Ostomy Wound Manage 61(3):28-33

6. Avitabile S, Odorisio T, Madonna S, Eyerich S, Guerra L, Eyerich K, Zambruno G, Cavani A, Cianfarani F (2015) Interleukin-22 promotes wound repair in diabetes by improving keratinocyte prohealing functions. J Invest Dermatol 135(11):2862-2870

7. Biondi B, Wartofsky L (2012) Combination treatment with T4 and T3: toward personalized replacement therapy in hypothyroidism? J Clin Endocrinol Metab 97(7):2256-2271

8. Bodo E, Kany B, Gaspar E, Knuver J, Kromminga A, Ramot Y, Biro T, Tiede S, van Beek N, Poeggeler B, Meyer KC, Wenzel BE, Paus R (2010) Thyroid-stimulating hormone, a novel, locally produced modulator of human epidermal functions, is regulated by thyrotropin-releasing hormone and thyroid hormones. Endocrinology 151(4):1633-1642 
9. Bodo E, Kromminga A, Funk W, Laugsch M, Duske U, Jelkmann W, Paus R (2007) Human hair follicles are an extrarenal source and a nonhematopoietic target of erythropoietin. FASEB J 21(12):3346-3354

10. Brown C, Stenn KS, Falk RJ, Woodley DT, O'Keefe EJ (1991) Vitronectin: effects on keratinocyte motility and inhibition of collagen-induced motility. J Invest Dermatol 96(5):724-728

11. Certan D, Righini V, Oliva M, Fioravanti P, Bevilacqua M (2013) Bioavailability of 1-thyroxine and its metabolites after topical treatment with an emulsion containing $0.1 \%$ micronised 1-thyroxine. G Ital Dermatol Venereol 148(3):287-292

12. Chen J, Ortmeier SB, Savinova OV, Nareddy VB, Beyer AJ, Wang D, Gerdes AM (2012) Thyroid hormone induces sprouting angiogenesis in adult heart of hypothyroid mice through the PDGF-Akt pathway. J Cell Mol Med 16(11):2726-2735

13. Cheng SY, Leonard JL, Davis PJ (2010) Molecular aspects of thyroid hormone actions. Endocr Rev 31(2):139-170

14. Cianfarani F, Baldini E, Cavalli A, Marchioni E, Lembo L, Teson M, Persechino S, Zambruno G, Ulisse S, Odorisio T, D'Armiento M (2010) TSH receptor and thyroid-specific gene expression in human skin. J Invest Dermatol 130(1):93-101

15. Demidova-Rice TN, Hamblin MR, Herman IM (2012) Acute and impaired wound healing: pathophysiology and current methods for drug delivery, part 1: normal and chronic wounds: biology, causes, and approaches to care. Adv Skin Wound Care 25(7):304-314

16. Deshayes N, Bloas F, Boissout F, Lecardonnel J, Paris M (2018) $3 \mathrm{D}$ In vitro model of the re-epithelialization phase in the woundhealing process. Exp Dermatol 27(5):460-462

17. Eming SA, Martin P, Tomic-Canic M (2014) Wound repair and regeneration: mechanisms, signaling, and translation. Sci Transl Med 6(265):265sr266. https://doi.org/10.1126/scitranslmed.30093 37

18. Eming SA, Tomic-Canic M (2017) Updates in wound healing: Mechanisms and translation. Exp Dermatol 26(2):97-98

19. Erdogan M, Ilhan YS, Akkus MA, Caboglu SA, Ozercan I, Ilhan N, Yaman M (1999) Effects of L-thyroxine and zinc therapy on wound healing in hypothyroid rats. Acta Chir Belg 99(2):72-77

20. Galiano RD, Michaels JT, Dobryansky M, Levine JP, Gurtner GC (2004) Quantitative and reproducible murine model of excisional wound healing. Wound Repair Regen 12(4):485-492

21. Gould L, Abadir P, Brem H, Carter M, Conner-Kerr T, Davidson J, DiPietro L, Falanga V, Fife C, Gardner S, Grice E, Harmon J, Hazzard WR, High KP, Houghton P, Jacobson N, Kirsner RS, Kovacs EJ, Margolis D, McFarland Horne F, Reed MJ, Sullivan DH, Thom S, Tomic-Canic M, Walston J, Whitney JA, Williams J, Zieman S, Schmader K (2015) Chronic wound repair and healing in older adults: current status and future research. J Am Geriatr Soc 63(3):427-438

22. Gurtner GC, Werner S, Barrandon Y, Longaker MT (2008) Wound repair and regeneration. Nature 453(7193):314-321

23. Hamed S, Bennett CL, Demiot C, Ullmann Y, Teot L, Desmouliere A (2014) Erythropoietin, a novel repurposed drug: an innovative treatment for wound healing in patients with diabetes mellitus. Wound Repair Regen 22(1):23-33

24. Haslam IS, Jadkauskaite L, Szabo IL, Staege S, Hesebeck-Brinckmann J, Jenkins G, Bhogal RK, Lim FL, Farjo N, Farjo B, Biro T, Schafer M, Paus R (2017) Oxidative damage control in a human (mini-) organ: Nrf2 activation protects against oxidative stressinduced hair growth inhibition. J Invest Dermatol 137(2):295-304

25. Heyer K, Herberger K, Protz K, Glaeske G, Augustin M (2016) Epidemiology of chronic wounds in Germany: analysis of statutory health insurance data. Wound Repair Regen 24(2):434-442

26. Heyer K, Protz K, Glaeske G, Augustin M (2017) Epidemiology and use of compression treatment in venous leg ulcers: nationwide claims data analysis in Germany. Int Wound J 14(2):338-343
27. Ilonzo N, Patel M, Lantis JC 2nd (2018) Managing the diabetic foot ulcer: how best practices fit the real 2018 United States. Surg Technol Int 32:49-59

28. Jimenez F, Poblet E, Izeta A (2015) Reflections on how wound healing-promoting effects of the hair follicle can be translated into clinical practice. Exp Dermatol 24(2):91-94

29. Kassem R, Liberty Z, Babaev M, Trau H, Cohen O (2012) Harnessing the skin-thyroid connection for wound healing: a prospective controlled trial in guinea pigs. Clin Exp Dermatol 37(8):850-856

30. Ketomaki T, Vahatupa M, May U, Pemmari T, Ruikka E, Hietamo J, Kaipiainen P, Barker H, Parkkila S, Uusitalo-Jarvinen H, Jarvinen TAH (2019) R-Ras regulates vascular permeability, but not overall healing in skin wounds. Exp Dermatol 28(2):202-206

31. Langan EA, Fink T, Paus R (2018) Is prolactin a negative neuroendocrine regulator of human skin re-epithelisation after wounding? Arch Dermatol Res 310(10):833-841

32. Laurent I, Tang S, Astere M, Wang KR, Deng S, Xiao L, Li QF (2018) Liquid L-thyroxine versus tablet L-thyroxine in patients on L-thyroxine replacement or suppressive therapy: a metaanalysis. Endocrine 61(1):28-35

33. Lebonvallet N, Laverdet B, Misery L, Desmouliere A, Girard D (2018) New insights into the roles of myofibroblasts and innervation during skin healing and innovative therapies to improve scar innervation. Exp Dermatol 27(9):950-958

34. Liao T, Lehmann J, Sternstein S, Yay A, Zhang G, Matthiessen AE, Schumann S, Siemers F, Kruse C, Hundt JE, Langan EA, Tiede S, Paus R (2019) Nestin(+) progenitor cells isolated from adult human sweat gland stroma promote reepithelialisation and may stimulate angiogenesis in wounded human skin ex vivo. Arch Dermatol Res 311(4):325-330

35. Lim YC, Bhatt MP, Kwon MH, Park D, Na S, Kim YM, Ha KS (2015) Proinsulin C-peptide prevents impaired wound healing by activating angiogenesis in diabetes. J Invest Dermatol 135(1):269-278

36. Liu X, Zheng N, Shi YN, Yuan J, Li L (2014) Thyroid hormone induced angiogenesis through the integrin alphavbeta3/protein kinase D/histone deacetylase 5 signaling pathway. J Mol Endocrinol 52(3):245-254

37. Lu Z, Hasse S, Bodo E, Rose C, Funk W, Paus R (2007) Towards the development of a simplified long-term organ culture method for human scalp skin and its appendages under serum-free conditions. Exp Dermatol 16(1):37-44

38. Martin P, Nunan R (2015) Cellular and molecular mechanisms of repair in acute and chronic wound healing. Br J Dermatol 173(2):370-378

39. Martinez ML, Escario E, Poblet E, Sanchez D, Buchon FF, Izeta A, Jimenez F (2016) Hair follicle-containing punch grafts accelerate chronic ulcer healing: a randomized controlled trial. J Am Acad Dermatol 75(5):1007-1014

40. Mecklenburg L, Tobin DJ, Muller-Rover S, Handjiski B, Wendt G, Peters EM, Pohl S, Moll I, Paus R (2000) Active hair growth (anagen) is associated with angiogenesis. J Invest Dermatol 114(5):909-916

41. Meier NT, Haslam IS, Pattwell DM, Zhang GY, Emelianov V, Paredes R, Debus S, Augustin M, Funk W, Amaya E, Kloepper JE, Hardman MJ, Paus R (2013) Thyrotropin-releasing hormone $(\mathrm{TRH})$ promotes wound re-epithelialisation in frog and human skin. PLoS ONE 8(9):e73596

42. Michaels JT, Churgin SS, Blechman KM, Greives MR, Aarabi S, Galiano RD, Gurtner GC (2007) db/db mice exhibit severe wound-healing impairments compared with other murine diabetic strains in a silicone-splinted excisional wound model. Wound Repair Regen 15(5):665-670

43. Moll I, Houdek P, Schmidt H, Moll R (1998) Characterization of epidermal wound healing in a human skin organ culture 
model: acceleration by transplanted keratinocytes. J Invest Dermatol 111(2):251-258

44. Olah A, Gherardini J, Bertolini M, Cheret J, Ponce L, Kloepper J, Biro T, Soeberdt M, Abels C, Paus R (2016) The thyroid hormone analogue KB2115 (eprotirome) prolongs human hair growth (anagen) ex vivo. J Invest Dermatol 136(8):1711-1714

45. Pastar I, Wong LL, Egger AN, Tomic-Canic M (2018) Descriptive vs mechanistic scientific approach to study wound healing and its inhibition: is there a value of translational research involving human subjects? Exp Dermatol 27(5):551-562

46. Patterson J (2015) Weedon's skin pathology, 4th edn. Elsevier

47. Paus R (2010) Exploring the "thyroid-skin connection": concepts, questions, and clinical relevance. J Invest Dermatol 130(1):7-10

48. Peplow PV, Chatterjee MP (2013) A review of the influence of growth factors and cytokines in in vitro human keratinocyte migration. Cytokine 62(1):1-21

49. Pomari E, Dalla Valle L, Pertile P, Colombo L, Thornton MJ (2015) Intracrine sex steroid synthesis and signaling in human epidermal keratinocytes and dermal fibroblasts. FASEB J 29(2):508-524

50. Pop MA, Almquist BD (2017) Biomaterials: a potential pathway to healing chronic wounds? Exp Dermatol 26(9):760-763

51. Qi L, Ahmadi AR, Huang J, Chen M, Pan B, Kuwabara H, Iwasaki K, Wang W, Wesson R, Cameron AM, Cui S, Burdick J, Sun Z (2020) Major improvement in wound healing through pharmacologic mobilization of stem cells in severely diabetic rats. Diabetes 69(4):699-712

52. Rotty JD, Coulombe PA (2012) A wound-induced keratin inhibits Src activity during keratinocyte migration and tissue repair. J Cell Biol 197(3):381-389

53. Safer JD (2013) Thyroid hormone and wound healing. J Thyroid Res 2013:124538

54. Safer JD, Crawford TM, Holick MF (2005) Topical thyroid hormone accelerates wound healing in mice. Endocrinology 146(10):4425-4430

55. Schiekofer S, Andrassy M, Chen J, Rudofsky G, Schneider J, Wendt T, Stefan N, Humpert P, Fritsche A, Stumvoll M, Schleicher E, Haring HU, Nawroth PP, Bierhaus A (2003) Acute hyperglycemia causes intracellular formation of CML and activation of ras, p42/44 MAPK, and nuclear factor kappaB in PBMCs. Diabetes 52(3):621-633

56. Schreml S, Szeimies RM, Prantl L, Karrer S, Landthaler M, Babilas P (2010) Oxygen in acute and chronic wound healing. Br J Dermatol 163(2):257-268

57. Siegenthaler W, Amann-Vesti B (2006) Klinische Pathophysiologie, 9 th edn. ISBN: 978-3-13-449609-3

58. Stenn KS (1981) Epibolin: a protein of human plasma that supports epithelial cell movement. Proc Natl Acad Sci USA 78(11):6907-6911

59. Stevenson S, Sharpe DT, Thornton MJ (2009) Effects of oestrogen agonists on human dermal fibroblasts in an in vitro wounding assay. Exp Dermatol 18(11):988-990

60. Straseski JA, Gibson AL, Thomas-Virnig CL, Allen-Hoffmann BL (2009) Oxygen deprivation inhibits basal keratinocyte proliferation in a model of human skin and induces regio-specific changes in the distribution of epidermal adherens junction proteins, aquaporin-3, and glycogen. Wound Repair Regen 17(4):606-616

61. Sullivan TP, Eaglstein WH, Davis SC, Mertz P (2001) The pig as a model for human wound healing. Wound Repair Regen $9(2): 66-76$
62. Takagi N, Kawakami K, Kanno E, Tanno H, Takeda A, Ishii K, Imai Y, Iwakura Y, Tachi M (2017) IL-17A promotes neutrophilic inflammation and disturbs acute wound healing in skin. Exp Dermatol 26(2):137-144

63. Tiede S, Bohm K, Meier N, Funk W, Paus R (2010) Endocrine controls of primary adult human stem cell biology: thyroid hormones stimulate keratin 15 expression, apoptosis, and differentiation in human hair follicle epithelial stem cells in situ and in vitro. Eur J Cell Biol 89(10):769-777

64. van Beek N, Bodo E, Kromminga A, Gaspar E, Meyer K, Zmijewski MA, Slominski A, Wenzel BE, Paus R (2008) Thyroid hormones directly alter human hair follicle functions: anagen prolongation and stimulation of both hair matrix keratinocyte proliferation and hair pigmentation. J Clin Endocrinol Metab 93(11):4381-4388

65. Vidali S, Cheret J, Giesen M, Haeger S, Alam M, Watson REB, Langton AK, Klinger M, Knuever J, Funk W, Kofler B, Paus R (2016) Thyroid hormones enhance mitochondrial function in human epidermis. J Invest Dermatol 136(10):2003-2012

66. Vidali S, Knuever J, Lerchner J, Giesen M, Biro T, Klinger M, Kofler B, Funk W, Poeggeler B, Paus R (2014) Hypothalamicpituitary-thyroid axis hormones stimulate mitochondrial function and biogenesis in human hair follicles. J Invest Dermatol 134(1):33-42

67. Walter MN, Wright KT, Fuller HR, MacNeil S, Johnson WE (2010) Mesenchymal stem cell-conditioned medium accelerates skin wound healing: an in vitro study of fibroblast and keratinocyte scratch assays. Exp Cell Res 316(7):1271-1281

68. Weitzel JM, Iwen KA (2011) Coordination of mitochondrial biogenesis by thyroid hormone. Mol Cell Endocrinol 342(1-2):1-7

69. Wen J, Li X, Leng X, Xu X, Wu X (2017) An advanced mouse model for human skin wound healing. Exp Dermatol 26(5):433-435

70. Windoffer R, Beil M, Magin TM, Leube RE (2011) Cytoskeleton in motion: the dynamics of keratin intermediate filaments in epithelia. J Cell Biol 194(5):669-678

71. Wojcik SM, Bundman DS, Roop DR (2000) Delayed wound healing in keratin 6a knockout mice. Mol Cell Biol 20(14):5248-5255

72. Wood JM, Decker H, Hartmann H, Chavan B, Rokos H, Spencer JD, Hasse S, Thornton MJ, Shalbaf M, Paus R, Schallreuter KU (2009) Senile hair graying: H2O2-mediated oxidative stress affects human hair color by blunting methionine sulfoxide repair. FASEB J 23(7):2065-2075

73. Xu Y, Sun J, Carter RR, Bogie KM (2014) Personalized prediction of chronic wound healing: an exponential mixed effects model using stereophotogrammetric measurement. J Tissue Viability 23(2):48-59

74. Zhang GY, Langan EA, Meier NT, Funk W, Siemers F, Paus $\mathrm{R}$ (2019) Thyroxine (T4) may promote re-epithelialisation and angiogenesis in wounded human skin ex vivo. PLoS ONE 14(3):e0212659

75. Zhou L, Zhang X, Paus R, Lu Z (2018) The renaissance of human skin organ culture: a critical reappraisal. Differentiation 104:22-35

Publisher's Note Springer Nature remains neutral with regard to jurisdictional claims in published maps and institutional affiliations. 


\section{Affiliations}

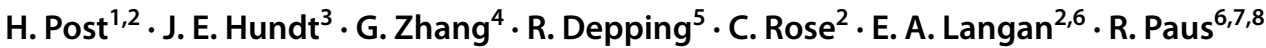

1 Department of Dermatology, University of Münster, Münster, Germany

2 Department of Dermatology, University of Lübeck, Lübeck, Germany

3 Lübeck Institute for Experimental Dermatology, Lübeck, Germany

4 Department of Plastic and Reconstructive Surgery, Shanghai Ninth People's Hospital, Shanghai, China

5 Institute of Physiology, University of Lübeck, Lübeck, Germany
6 Centre for Dermatology Research, University of Manchester and NIHR Manchester Biomedical Research Centre, Manchester, UK

7 Dr. Phillip Frost Department of Dermatology and Cutaneous Surgery, University of Miami Miller School of Medicine, Miami, FL, USA

8 Monasterium Laboratories, Münster, Germany 